\title{
Magnetic Properties of Gadolinium-Doped Amorphous Silicon Films
}

\author{
M.S. Sercheli, C. Rettori, \\ Instituto de Física Gleb Wataghin, Unicamp, 13.081-970 Campinas, SP, Brazil \\ and A.R. Zanatta \\ Instituto de Física de São Carlos-USP, 13560-250 São Carlos, SP, Brazil
}

Received on 23 April, 2001

\begin{abstract}
Electron spin resonance (ESR) and $d c$-magnetization experiments have been performed in Gd-doped amorphous $(a-)$ silicon films. The films were deposited by the cosputtering technique following different conditions rendering samples with varying $\mathrm{Gd}$ and hydrogen concentrations. In addition to films with different contents of impurities and, in order to probe the influence of the atomic structure on the magnetic properties of the Gd species, the films were also submitted to laser-induced crystallization processing. Both ESR and dc-magnetization results show that Gd is incorporated as a trivalent ion $\left(\mathrm{Gd}^{3+}\right)$ in the $\mathrm{Si}$ host. ESR data indicate a strong dependence between the $\mathrm{Gd}$ concentration and the density of Si dangling bonds. Moreover, the $\mathrm{Gd}^{3+}$ local environment is nearly insensitive to the Gd and $\mathrm{H}$ content, as well as to the atomic structure of the Si host. Along with gadolinium, other rare-earth species have been investigated and the main results are discussed.
\end{abstract}

Much of the current interest in studying rare-earth (RE) doped silicon-based compounds arises from their potential to combine the unique optical characteristics of $\mathrm{RE}^{3+}$ ions with the electrical properties of semiconductor hosts. Moreover, despite the great advances achieved in this area, still there are a lot of interesting and unanswered questions.[1] While various groups around the world succeeded in doping several (either crystalline or amorphous) Si-based matrices with different $\mathrm{RE}^{3+}$ ions, it is not yet completely clear what is the local chemical environment around the $\mathrm{RE}^{3+}$ ions and the involved energies.[2] Although it seems to be more an academic than a technological problem, it is important to stress that these two aspects may determine both, the design and the final performance of the desired opto-eletronic devices.

Based on these ideas this work presents a systematic and preliminary study of the magnetic properties of different series of Gd-doped amorphous $\left(a^{-}\right)$Si films prepared by the cosputtering technique. Gd atoms and the cosputtering technique have been respectively chosen in view of their magnetic characteristics and versatility in producing films with quite different and controllable atomic compositions. To get further insight, auxiliary spectroscopic techniques were employed to investigate the films. Besides, and were applicable, allusion to the study of other $\mathrm{RE}^{3+}$ ions in similar Si hosts is made.

Gd-doped $a$-Si and $a$-Si:H films have been deposited by the cosputtering technique in a standard radio frequency (13.56 MHz) system. The films, typically $\approx 1 \mu \mathrm{m}$ thick, were deposited on high-purity quartz substrates at $\approx 70^{\circ} \mathrm{C}$ in a $\mathrm{Ar}+\mathrm{H}_{2}$ atmosphere according to different target composition [Gd-to-Si relative area $\left.\left(\mathrm{A}_{G d} / \mathrm{A}_{S i}\right)\right]$ and partial pressures of hydrogen $\left[\mathrm{P}\left(\mathrm{H}_{2}\right)\right]$. After deposition, some of the Gd-doped $a_{-}$ $\mathrm{Si}(\mathrm{H})$ were crystallized with a Nd-YAG laser $(532.0 \mathrm{~nm}$ and pulse width of $10 \mathrm{~ns}$ ).[3] The films were investigated by means of optical (absorption and Raman scattering) techniques at room temperature. The ESR experiments were carried out in a Bruker X-band spectrometer, using a $\mathrm{TE}_{102}$ room- $T$ cavity and a helium gas flux $(4.2$ $\mathrm{K}-300 \mathrm{~K})$ temperature controller. $d c$-magnetization measurements were performed in a Quantum Design SQUID MPMS magnetometer (RSO mode). The sample was kept at the center of the scan range using the sample autotracking option.

The Gd concentration of the samples reported in this work were obtained from: $i$ ) Rutherford backscatering spectroscopy (RBS), $i i$ ) an estimation of the ESR signal intensity as compared with one ESR standard, and iii) a Curie-Weiss fitting of the low-temperature $d c$-magnetic susceptibility, $\chi(T)$, data, after substraction of the diamagnetism of a similar undoped thin film/quartz substrate sample. The hydrogen content of the films were determined by either Nuclear reaction analysis (NRA) or optical absorption in the infrared region. Some of the main characteristics of the studied $\mathrm{Gd}$-doped $a$-Si(H) films are presented in Table I.

The temperature dependence of the $[\chi(T)](1 \mathrm{~T})$ were measured for all $a$-Si(H) films, and the data was fitted to a Curie-Weiss law with different $\mathrm{Gd}^{3+}\left(4 f^{7}\right.$, $\left.S=7 / 2,7.94 \mu_{B}\right)$ concentration values. The estimated 
$\mathrm{Gd}^{3+}$ concentration and paramagnetic temperature $[\theta]$ for all the studied films are given in Table I. The ESR spectra consist of a broad and single resonance line associated to a powder-like spectrum of $\mathrm{Gd}^{3+}$. The intensity of this resonance depends on [Gd] and increases at low temperature following approximately the $1 / T$ behaviour appropriate for localized magnetic moments. Within the experimental error, the $g$-value and linewith $\Delta H_{p p}$ does not depend on the $\mathrm{Gd}^{3+}$ concentration and temperature for $T \gtrsim 30 \mathrm{~K}$. On the other hand, Gd-doped $a$-Si films with [Gd] $\gtrsim 4 a t . \%$ and $T \lesssim$ $30 \mathrm{~K}$, present a small line broadening, suggesting the existence of $\mathrm{Gd}-\mathrm{Gd}$ magnetic interactions. However, low field $0.1-0.5 \mathrm{kG}$ susceptibility measurements in these high concentrated films did not show any spinglass behavior. [4] The $\mathrm{Gd}^{3+}$ concentration was also determined comparing the ESR intensity with a known $\mathrm{Gd}_{1.45} \mathrm{Ce}_{0.55} \mathrm{RuSr}_{2} \mathrm{Cu}_{2} \mathrm{O}_{10+\delta}$ sample and a strong $\mathrm{KCl}-$ pitch standard. The main ESR parameters and $\mathrm{Gd}^{3+}$ concentration are given in Table I. The values obtained from the $\chi(T)$ measurements and the density of Si dangling bonds are also shown in Table I.

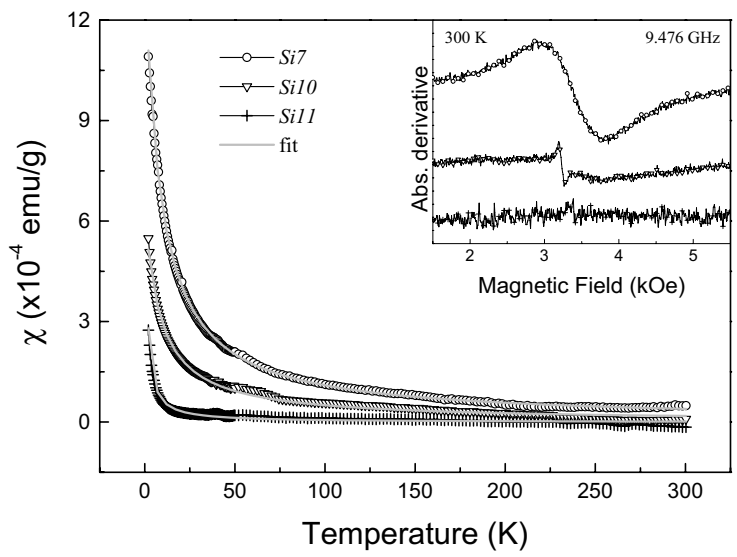

Figure 1. $\chi(T)$ of the hydrogenated films Si7, Si10 and Si11, at $1 \mathrm{~T}$, after subtraction of the pure $a$-Si film/quartz substrate diamagnetism. Continuous lines correspond to a Curie-Wiess law and the fitting parameters are given in Table I. Inset: room temperature ESR spectra of the same Gd-doped $a$-Si films with varying $[\mathrm{H}]$.

The $\chi(T)$ of the (hydrogenated) Si7, Si10, and Si11 films, measured at $1 \mathrm{~T}$, are represented in Figure1. The experimental data was fitted to a Curie-Weiss law with $4.7,1.9$, and 0.2 at. $\%$ of $\mathrm{Gd}^{3+}\left(4 f^{7}, S=7 / 2,7.94 \mu_{B}\right)$ for samples Si7, Si10, and Si11, respectively. The inset of Fig. 1 shows the room temperature ESR spectra of the same hydrogenated films. ESR measurements were also performed at low temperatures and consist of a broad and single resonance line that, within the experimental error, does not depend on $[\mathrm{H}]$ nor on the temperature. Similarly to the non-hydrogenated films, the intensity of this resonance signal increases at low temperatures and follows approximately the $1 / T$ behavior. The magnetic characteristics of these samples are also shown in Table I.

Notice that, despite the use of a constant $\mathrm{A}_{G d} / \mathrm{A}_{S i}$, the presence of $\mathrm{H}_{2}$ during deposition reduces the final concentration of $\mathrm{Gd}$ in the films. It is an expected result since we have adopted a constant total pressure of $5 \times 10^{-3}$ Torr for all deposition runs. As a consequence, and taking into account the sputter yield due to $\mathrm{H}_{n}^{+}$and $\mathrm{Ar}^{+}$ions, [5] the concentration of $\mathrm{Gd}$ will be smaller as more and more hydrogen atoms are used during the depositions.

The effect of hydrogen insertion in the $a$-SiGd matrix was also investigated through optical spectroscopy. As expected,[6] and according to the experimental data, the introduction of hydrogen in the $a$-SiGd matrix induces a bandgap widening and a concomitant passivation of Si dangling bonds (see Table I).

At this point it is important to notice that, while hydrogen atoms promote a real passivation of the Si dangling bonds, the $\mathrm{Gd}^{3+}$ ions act in a different manner in reducing the $[d b]$. The density of Si dangling bonds, as obtained from the ESR measurements for various different RE-doped (RE = Y, La, Gd, Er, and Lu) $a$-Si films, is strongly suppressed only by the magnetic $\mathrm{RE}^{3+}$ ions.[7] This indicates that $\mathrm{RE}^{3+}$ ion size effects can be neglected as a possible $d b$ depleting mechanism, and the spin component of the $\mathrm{RE}^{3+}$ (maximum for $\mathrm{Gd}^{3+}$ ) is probably the main responsible for the $[d b]$ diminution.

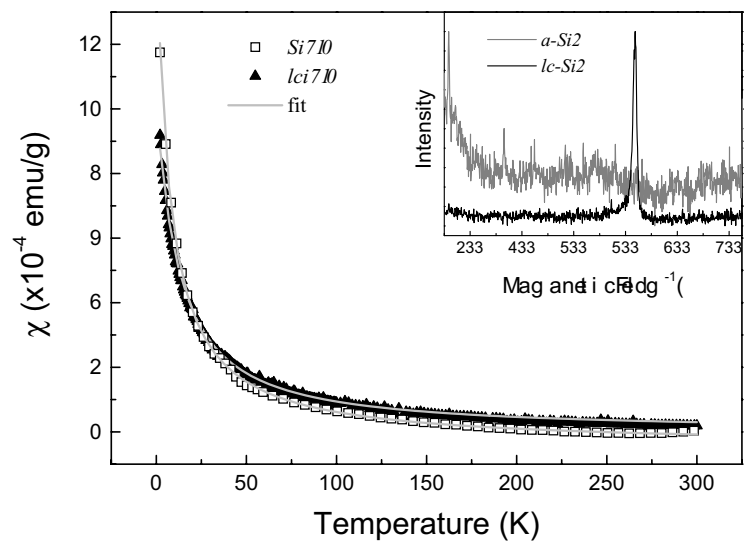

Figure 2. dc-magnetization susceptibility, at $1 \mathrm{~T}$, of film Si2 before $(a$-Si2 $)$ and after $(l c-\mathrm{Si} 2)$ laser-crystallization experiments. The Curie-Wiess law is also represented for $\chi(\mathrm{T})$ and the fitting parameters are given in Table I. Inset: room temperature Raman scattering spectra of these same films. After laser treatment, notice the appearance of a Raman signal at $\approx 520 \mathrm{~cm}^{-1}$ due to the existence of Si nano-crystals.

Some results of the laser-crystallization experiments are presented in Figure 2 where the $\chi(\mathrm{T})$ spectra, measured at $1 \mathrm{~T}$, of film $\mathrm{Si} 2$ are shown before and after crystallization. The inset displays the corresponding room temperature Raman spectra for the same films. As can be seen from the inset, the asgrown film presents a very weak and almost featureless spectrum characteristic of an amorphous quasi-metallic 
compound.[8] After laser-crystallization, the film exhibits a well defined Raman signal at $~ 520 \mathrm{~cm}^{-1}$ typical of Si nano-crystals.

Except for a partially crystallized atomic structure,[9] the experimental data was fitted to a Curie-Weiss law with 6 at. $\%$ of $\mathrm{Gd}^{3+}$, and the magnetic characteristics of the laser-crystallized films resemble very much those achieved in the amorphous case, i.e., before the laser-induced crystallization processing.

Based on the above experimental results the ESR and $d c$-magnetic susceptibility data demonstrate unambiguously that $\mathrm{Gd}$ atoms are incorporated in Si as trivalent ions $\mathrm{Gd}^{3+}$. Moreover, the ESR results show that the $\mathrm{Gd}^{3+}$ powder-like spectra parameters are not sensitive to the $\mathrm{Gd}$ concentration, atomic structure, and $\mathrm{H}$ content. Both the $g$-value and linewidth for all the studied films are about the same (Table I). This is an intriguing result, because it is known that the $\mathrm{Gd}^{3+}$ ESR spectra is composed of an anisotropic fine structure due to crystal field effects and, as a consequence, it should be affected by different environments at the $\mathrm{Gd}^{3+}$ sites. These results suggest that the neighborhood of the $\mathrm{RE}^{3+}$, in $a$-Si hosts, is not affected by the different atomic structure of the studied films. A possible reason for such a behavior could be the formation of a very stable covalent-like $\mathrm{RESi}_{2}$ compound.

Thin films of Gd-doped $a-\mathrm{Si}: \mathrm{H}$, with different [Gd] and $[\mathrm{H}]$, have been prepared by the cosputtering technique. Allied with some optical properties, the $\chi(T)$ and ESR results presented in this work allowed us to conclude that: $i$ ) Gd atoms are incorporated as trivalent ions $\left(\mathrm{RE}^{3+}\right)$ in $a$-Si matrices, $\left.i i\right)$ the $\mathrm{RE}^{3+}$ ions incorporated in our films form an extremely stable complex, probably $\mathrm{RESi}_{2}$ and/or some related covalent compound, iii) in the $a$-SiRE:H films the actual RE concentration is much smaller than the expected one (based on $\mathrm{A}_{G d} / \mathrm{A}_{S i}$ ) as a consequence of the deposition method, $i v)$ the $[\mathrm{db}]$ in the $a-\mathrm{Si}$ films is strongly depleted by the magnetic $\mathrm{RE}^{3+}$ species. The strongest effect is obtained for $\mathrm{Gd}^{3+}$, suggesting a mechanism associated to the spin component of the $\mathrm{RE}^{3+}$ via a strong exchange field-like coupling between the $\mathrm{RE}^{3+}$ and $\mathrm{db}$ spins.

The authors are indebted to Professor F.L. Freire Jr. (PUC-RJ) for the ion beam analyses and to Professor F. Iikawa (UNICAMP) for the access to the Raman facilities. This work was supported by CAPES-Brazil, CNPq-Brazil, FAPESP-Brazil, NSF-DMR-USA, and NSF-INT-USA.

Table I - Gd concentration as determined from ESR (room temperature) and $\chi(T)$ measurements. The $g$-value, $\Delta H_{p p}$, Si dangling bond density $[d b], \theta$ values and the atomic concentration of $\mathrm{H}$ and $\mathrm{Gd}$, as determined from ion beam analyses (NRA and RBS), are also displayed for the series of Gd-doped a-Si:H films.

\begin{tabular}{ccccccccc}
\hline Sample & {$[\mathrm{Gd}]_{E S R}$} & {$[\mathrm{Gd}]_{\chi}$} & g-value & $\Delta H_{p p}$ & {$[d b]$} & $\theta$ & {$[\mathrm{H}]_{N R A}$} & {$[\mathrm{Gd}]_{R B S}$} \\
\hline$\#$ & $($ at.\%) & $($ at. $\%)$ & - & $(\mathrm{Oe})$ & $\mathrm{cm}^{-3}$ & $(\mathrm{~K})$ & $($ at.\%) & $($ at.\%) \\
Si1 & 7.6 & $10(2)$ & $2.00(4)$ & $860(100)$ & $5 \times 10^{16}$ & $-12(2)$ & 1 & 7.5 \\
Si2 & 5.7 & $6(1)$ & $2.00(4)$ & $830(100)$ & $1.0 \times 10^{17}$ & $-7(2)$ & 1 & 4.0 \\
Si3 & 3.8 & $4.8(9)$ & $2.01(4)$ & $790(100)$ & $2.5 \times 10^{17}$ & $-7(2)$ & 1 & 2.5 \\
Si4 & 1.4 & $1.9(4)$ & $2.03(4)$ & $800(100)$ & $1.5 \times 10^{18}$ & $-4(1)$ & 1 & 1.5 \\
Si5 & 1.5 & $1.3(4)$ & $2.02(4)$ & $850(100)$ & $1.7 \times 10^{18}$ & $-3(1)$ & 1 & 1.0 \\
Si6 & 1.3 & $0.5(1)$ & $1.99(4)$ & $750(100)$ & $3.4 \times 10^{18}$ & $-4(1)$ & 1 & 0.5 \\
Si7 & 4.7 & $4.7(9)$ & $2.01(4)$ & $860(100)$ & $3.0 \times 10^{17}$ & $-9(2)$ & 1.2 & 2.5 \\
Si8 & 2.7 & $3.9(8)$ & $2.01(4)$ & $800(100)$ & $1.0 \times 10^{17}$ & $-6(2)$ & 2.7 & 2.0 \\
Si9 & 1.1. & $4.4(9)$ & $2.03(4)$ & $1020(100)$ & $5.0 \times 10^{16}$ & $-6(2)$ & 3.7 & 2.3 \\
Si10 & 1.2 & $1.9(5)$ & $2.07(4)$ & $1000(100)$ & $1.0 \times 10^{17}$ & $-8(2)$ & 7.5 & 1.5 \\
Si11 & - & $0.2(1)$ & - & - & $5.0 \times 10^{16}$ & $-0.4(0.5)$ & 9.0 & 0.4 \\
\hline
\end{tabular}

\section{References}

[1] Rare-Earth Doped Semiconductors II, Eds. S. Coffa, A. Polman, and R. Schwartz (Mater. Res. Soc., Pittsburgh, 1996), Vol. 422.

[2] For a review on the subject see, for instance, A. Polman, J. Appl. Phys. 82, 1 (1997).

[3] See, for example, M. Bell, L. Nunes, and A.R. Zanatta, J. Appl. Phys. 86, 701 (1999).

[4] F. Hellman, M.Q. Tran, A.E. Gebala, E.M. Wilcox, and R.C. Dynes, Phys. Rev. Lett. 77, 4652 (1996).
[5] B. Chapman, in Glow Discharge Processes, Sputtering and Plasma Etching (John Wiley, NY, 1980).

[6] R.A. Street, in Hydrogenated Amorphous Silicon (Cambridge University Press, Cambridge 1991).

[7] M.S. Sercheli, A.R. Zanatta, and C. Rettori, Magnetic Properties of Rare Earth Doped Amorphous Silicon Films (to be publish).

[8] J.S. Lannin, in Semiconductors and Semimetals, Ed. J.I. Pankove (Academic, NY, 1984), Vol. 21B, pp. 159-196.

[9] Z. Iqbal and S. Veprek, J. Phys. C: Solid State Phys. 15, 377 (1982). 